\title{
Cardiovascular Safety of Empagliflozin Versus Dipeptidyl Peptidase-4 (DPP-4) Inhibitors in Type 2 Diabetes: Systematic Literature Review and Indirect Comparisons
}

\author{
Chakrapani Balijepalli - Rohan Shirali • Prashanth Kandaswamy • \\ Anastasia Ustyugova · Egon Pfarr · Søren S. Lund · Eric Druyts
}

Received: May 4, 2018 / Published online: June 12, 2018

(C) The Author(s) 2018

\begin{abstract}
Introduction: Clinical trials conducted in patients with type 2 diabetes (T2DM) treated with glucose-lowering drugs and examining cardiovascular-related outcomes have yielded mixed results. In this work, we aimed to assess the relative treatment effects of empagliflozin versus sitagliptin and saxagliptin (dipeptidyl peptidase-4 (DPP-4) inhibitors) on cardiovascular-related outcomes in patients with T2DM.

Methods: We conducted a systematic literature review to identify clinical trials assessing cardiovascular-related outcomes for sitagliptin-, saxagliptin-, and empagliflozin-treated patients with T2DM. A network meta-analysis of indirect treatment comparisons was conducted in a Bayesian framework. Hazard ratios (HR) and 95\% credible intervals (CrI) were computed for
\end{abstract}

Enhanced digital features To view enhanced digital features for this article go to https://doi.org/10.6084/ m9.figshare.6391322.

Electronic supplementary material The online version of this article (https://doi.org/10.1007/s13300018-0456-7) contains supplementary material, which is available to authorized users.

C. Balijepalli $(\bowtie) \cdot$ R. Shirali $\cdot$ E. Druyts

Precision Health Economics, Vancouver, BC,

Canada

e-mail: chakrapani.balijepalli@precisionxtract.com

P. Kandaswamy · A. Ustyugova - E. Pfarr · S. S. Lund Boehringer Ingelheim GmbH, Ingelheim, Germany six cardiovascular-related outcomes to estimate the relative efficacies of these agents.

Results: Empagliflozin showed a statistically significant superiority over saxagliptin (HR 0.60 ; 95\% CrI 0.46-0.80) and sitagliptin (HR $0.60 ; 95 \%$ CrI $0.46-0.79$ ) to reduce the risk for cardiovascular-related mortality. For all-cause mortality, empagliflozin showed a statistically significant risk reduction compared to saxagliptin (HR 0.61; 95\% CrI 0.49-0.76) and sitagliptin (HR 0.67; 95\% CrI 0.54-0.83). A similar pattern was observed in the risk reduction for hospitalization due to heart failure, where empagliflozin was found to be statistically significantly superior to saxagliptin (HR $0.51 ; 95 \%$ CrI 0.37-0.70) and sitagliptin (HR 0.65; 95\% CrI 0.47-0.90). Empagliflozin was not statistically significantly different to sitagliptin and saxagliptin with regard to the risk of a composite endpoint composed of death, stroke or myocardial infarction.

Conclusion: In this indirect comparison to the DPP-4 inhibitors saxagliptin and sitagliptin, empagliflozin significantly lowered the risk of cardiovascular-related mortality, all-cause mortality and hospitalizations due to heart failure.

Funding: Boehringer Ingelheim GmbH.

Keywords: Cardiovascular outcomes; DPP-4 inhibitors; Empagliflozin; Network metaanalysis; Saxagliptin; Sitagliptin 


\section{INTRODUCTION}

Type 2 diabetes (T2DM) impacts one in every eleven adults, with a global prevalence of $8.8 \%$ ( $\sim 425$ million people) in the age group 20-79 years, according to the latest estimates of the International Diabetes Federation (IDF). Current evidence suggests that the number of people with diabetes will increase to 629 million in that age group by the year 2045 [1]. Individuals impacted by T2DM have a substantially elevated risk for cardiovascular (CV) events; the risk of cardiovascular disease is two to four times higher in diabetics compared to nondiabetics [2-5]. Hospital discharge data from 2014 in the United States (US) shows diabetes as a medical diagnosis in 7.2 million US hospital discharges, with 1.5 million of those discharges reporting major comorbid CV diagnoses (70.4 per 1000 persons with diabetes) [6]. The importance of preventing cardiovascular complications while managing patients with T2DM has been emphasized in position statements issued by the American Diabetes Association (ADA) and the European Association for the Study of Diabetes (EASD) [7, 8]. Previous clinical trials that were designed to examine cardiovascular-related events in patients undergoing intensive glycaemic control have shown mixed results, and uncertainty remains over the impact of glycaemic control on macrovascular event reduction in people with diabetes, whereas a possible favourable impact of intensive glucose control on microvascular outcomes seems to have a broader consensus [9]. One key area of concern is whether the use of certain classes of antihyperglycaemic agents are associated with an increased risk of cardiovascular outcomes [10]. As a result, international regulatory agencies require that newer antihyperglycaemic agents must demonstrate not only their efficacy in glycaemic control but also that they do not significantly increase the risk of cardiovascular events in patients with T2DM $[11,12]$.

In general, results from large-scale randomized controlled trials (RCTs) designed specifically to assess long-term cardiovascular-related outcomes in patients treated with glucose- lowering drugs have provided mixed results. The Empagliflozin, Cardiovascular Outcomes, and Mortality in Type 2 Diabetes (EMPA-REG OUTCOME) trial was a dedicated cardiovascular outcomes trial (CVOT) in patients with type 2 diabetes and established cardiovascular disease according to the Food and Drug Administration (FDA) 2008 guidance. In that trial, patients allocated to empagliflozin had a significantly lower risk of the primary composite cardiovascular outcome (CV death, stroke, or myocardial infarction), all-cause death, hospitalizations for heart failure and cardiovascular-related death compared to patients who were allocated placebo [13]. Since the FDA's 2008 guidance document was published, several other trials investigating other glucose-lowering treatments have been conducted and subsequently met the FDA's CV safety criteria, but individual cardiovascular outcomes such as heart failure have yielded inconsistent results [14-17].

Newer classes of glucose-lowering treatments have proven their cardiovascular benefit in T2DM patients, but these treatments have never been compared head-to-head in terms of their cardiovascular safety. Therefore, the objective of this study was to conduct a systematic literature review of RCTs evaluating long-term cardiovascular outcomes in T2DM patients treated with empagliflozin or dipeptidyl peptidase-4 (DPP-4) inhibitors, and to use network meta-analysis (NMA) to indirectly assess their relative cardiovascular effects.

\section{METHODS}

\section{Systematic Literature Review}

\section{Eligibility Criteria}

For this review, we examined cardiovascular outcomes assessed in studies conducted in adult patients with T2DM with established cardiovascular disease or at a high risk of experiencing a cardiovascular outcome. We gathered evidence to evaluate empagliflozin, a sodium-glucose co-transporter-2 (SGLT-2) inhibitor, in comparison with the class of DPP-4 inhibitors (alogliptin, linagliptin, saxagliptin, sitagliptin, vildagliptin). The complete study eligibility 
criteria, defined according to the populations, interventions, comparators, outcomes, and study design (PICOS) approach, are provided in Table S1 of the Electronic supplementary material (ESM).

\section{Search Strategy}

A comprehensive systematic literature search of the Medical Literature Analysis and Retrieval System Online (MEDLINE), Excerpta Medica Database (EMBASE), and the Cochrane Central Register of Controlled Trials was conducted on November 30, 2016. Additionally, manual searches of clinicaltrials.gov and conference proceedings of major diabetes conferences for the past two years were performed to identify RCTs that had not been published in a full manuscript but were potentially eligible for inclusion. Tables $\mathrm{S} 2-\mathrm{S} 4$ of the ESM provide the detailed search strategies.

\section{Study Selection and Data Extraction}

Two investigators independently reviewed all abstracts and proceedings identified in the literature search. All studies identified as eligible during the abstract screening were screened at the full-text stage by the same two reviewers. At each stage, if any discrepancies occurred between the studies that the two investigators selected, a third investigator provided arbitration. The two investigators independently extracted data on patient and study characteristics, including demographic information on patients (i.e. age, sex, race, weight), patient risk factors (i.e. cholesterol, blood pressure and liver function), cardiovascular comorbidities (i.e. prior stroke, prior myocardial infarction, coronary artery disease, prior heart failure), concomitant treatments (i.e. antihypertensives and cholesterol-lowering drugs), and diabetes progression factors (i.e. disease duration, HbA1c levels, concomitant antidiabetic medication).

Hazard ratios (HRs) for time-to-event data were extracted for the following outcomes: cardiovascular-related mortality, all-cause mortality, nonfatal myocardial infarction, nonfatal stroke, hospitalization for heart failure, and the composite of cardiovascular mortality, nonfatal stroke and nonfatal myocardial infarction. Two reviewers independently extracted all data from the included studies and recorded the data in a spreadsheet. A third reviewer checked all data extraction. This article is based on previously conducted studies and does not contain any studies with human participants or animals performed by any of the authors.

\section{Statistical Analyses}

A Bayesian NMA was conducted to indirectly compare treatment effects of empagliflozin to those of the included DPP-4 inhibitors for each of the cardiovascular-related outcomes. The methodology follows the guidelines set out by the National Institute for Health and Care Excellence (NICE) Decision Support Unit, and employs a generalized linear model framework [18]. Each trial reports the HRs of the active treatment relative to placebo for each outcome of interest. In the NMA, the logarithms of these reported HRs are modelled as mean treatment effects, using a normal likelihood and an identity link, and indirect comparisons are modelled as the differences in estimated treatment effects.

The validity of NMA relies on a number of assumptions. Each assumption was assessed prior to conducting the analyses. These assumptions include (1) similarity of trials; (2) homogeneity of results, and (3) consistency between direct and indirect evidence. As part of the process of ensuring a valid NMA, an evaluation of the heterogeneity of patient demographic characteristics, cardiovascular risk factors, and diabetic disease progression was undertaken. While some heterogeneity is expected, not all prognostic factors (i.e. factors affecting the absolute outcome risk) are expected to also affect comparative effect estimates between treatments.

Results from the analyses are presented in Fig. 3 and Table S9 of the ESM. The primary output is the indirectly estimated HR of empagliflozin relative to each other intervention and its associated 95\% credible interval (CrI: a Bayesian analogue to the common confidence interval). The graphical representations employed are similar to forest plots in that they present HRs for all comparisons, with 
horizontal lines showing the 95\% CrIs. A dotted vertical line shows the point at which the treatments are equivalent; values to the left of this line are favourable for empagliflozin, while values to the right favour the comparator. As the outcome data presented in the source publications were intention-to-treat data, and as on-treatment data were not available from the included trials, a per-protocol analysis could not be performed.

Parameters of the different models were estimated using a Markov chain Monte Carlo (MCMC) method implemented in the OpenBUGS software package [19, 20]. Model convergence was assessed by the Gelman-Rubin statistic and visual examination of diagnostic plots. Prior distributions were uninformative. All analyses were performed using $\mathrm{R}$ version 3.0.3 (http://www.r-project.org/) and OpenBUGS version 3.2.3 (OpenBUGS Project Management Group).

\section{RESULTS}

\section{Evidence Base}

A systematic literature review was performed to identify publications relevant to NMA of surrogate outcomes and cardiovascular outcomes. The trial selection process is displayed in Fig. 1 (based on searches run on November 30, 2016), from an initial screening of 15,732 articles to the inclusion of three trials in this NMA for cardiovascular outcomes among patients treated for T2DM [13, 14, 16]. Four trials met eligibility for inclusion, but only three trials were included in the final NMA. The excluded trial, Examination of Cardiovascular Outcomes with Alogliptin Versus Standard of Care (EXAMINE), had a specific inclusion criterion-that a patient must have had an acute coronary syndrome within 15-90 days before randomizationwhich could not be verified among the other trials of interest [17]. While EMPA-REG OUTCOME and the Saxagliptin Assessment of Vascular Outcomes Recorded in Patients with Diabetes Mellitus (SAVOR)-Thrombolysis in Myocardial Infarction (TIMI) 53 trials excluded patients with an acute cardiovascular event within two months prior to enrolment in the study $[13,16]$. Since this discrepancy in baseline risk could not be adequately accounted for statistically, the EXAMINE trial was excluded to ensure valid analyses. In the treatments included in this analysis, drugs belonging to the class of DPP-4 inhibitors (saxagliptin and sitagliptin) and the SGLT-2 inhibitor empagliflozin were present. The quality of the included studies was assessed using the Cochrane risk of bias tool, and is presented in Tables S5a-c of the ESM; overall, the studies included were of high quality, with a low risk of bias.

Patient characteristics from the included trials are presented in Tables S6-S8 of the ESM. Mean age ranged from 63.1 years in EMPA-REG OUTCOME to 65.5 years in the Trial Evaluating Cardiovascular Outcomes with Sitagliptin (TECOS). The proportion of female patients ranged from $28.0 \%$ in EMPA-REG OUTCOME to $33.4 \%$ in SAVOR-TIMI 53. Caucasian patients were well represented, ranging from $67.6 \%$ in TECOS to $75.4 \%$ in SAVOR-TIMI 53; the proportion of Asian patients ranged from $10.8 \%$ in SAVOR-TIMI 53 to $22.6 \%$ in TECOS. Mean body-mass index (BMI) ranged from $30.2 \mathrm{~kg} / \mathrm{m}^{2}$ in TECOS to $31.2 \mathrm{~kg} / \mathrm{m}^{2}$ in SAVOR-TIMI 53. While EMPA-REG OUTCOME and TECOS included patients who had an established CVD, SAVOR-TIMI 53 also included primary prevention patients. There were no substantial differences in lipid parameters and blood pressure across the trials.

The included studies consistently reported comorbidities and concomitant cardiovascular medications of patients, which demonstrated modest levels of heterogeneity in reported values across studies. The lowest proportion of patients with prior myocardial infarction was reported in SAVOR-TIMI 53 (37.6\%), whereas EMPA-REG OUTCOME reported the highest proportion (46.7\%). EMPA-REG OUTCOME reported $23.1 \%$ of patients with a history of prior stroke, TECOS reported $24.6 \%$, and SAVOR-TIMI 53 did not include data on prior stroke. Reported percentages of coronary artery disease (CAD) ranged from $51.9 \%$ in TECOS to $78.7 \%$ in SAVOR-TIMI 53. Reported percentages of patients with a history of heart failure ranged from $9.9 \%$ in EMPA-REG OUTCOME to $18.3 \%$ 

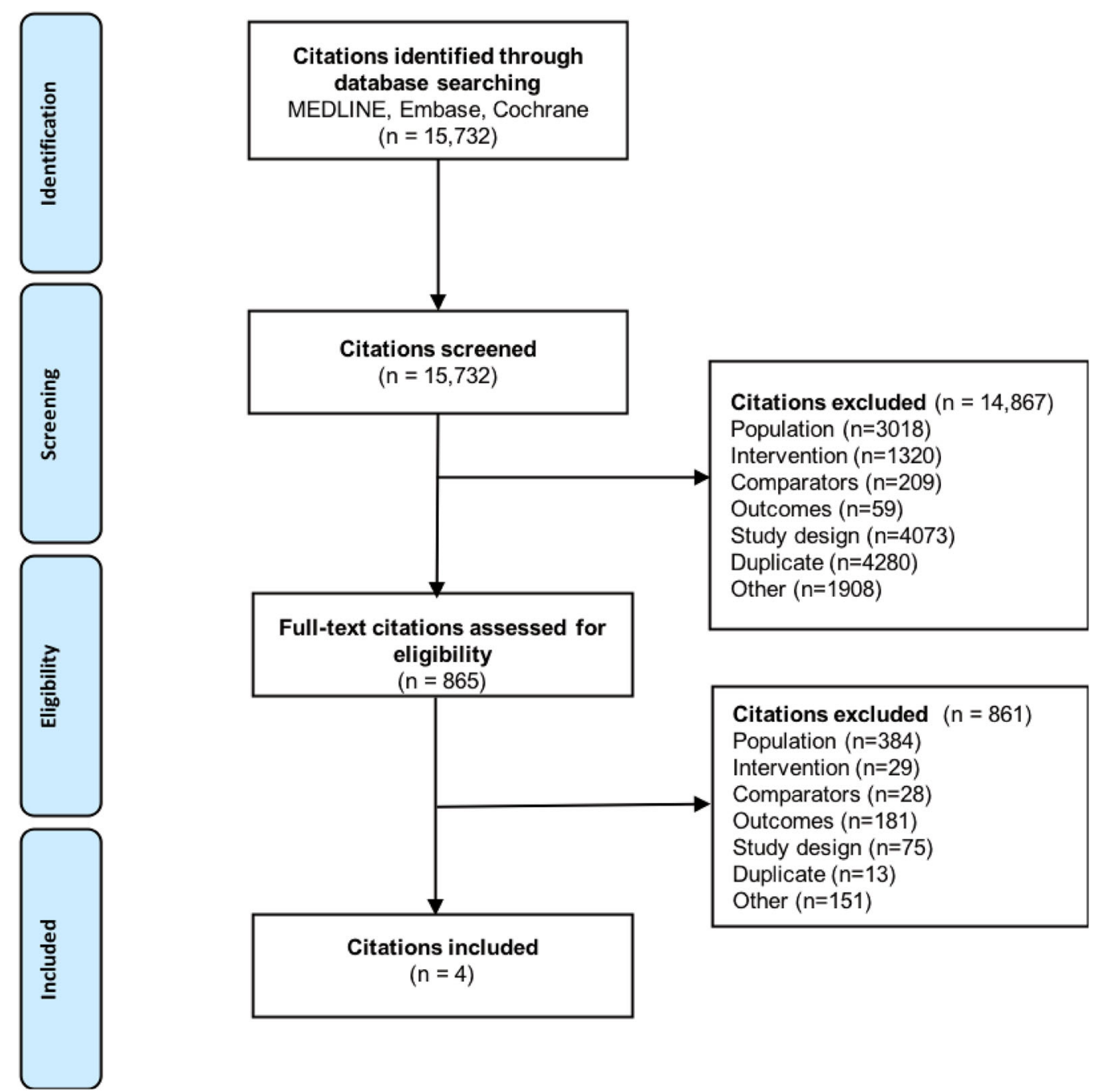

Fig. 1 PRISMA flow diagram

in TECOS. Use of beta-blockers and angiotensinconverting enzyme inhibitors (ACEis) ranged from $61.6 \%$ in SAVOR-TIMI 53 to $65.2 \%$ in EMPA-REG OUTCOME for beta-blockers, and from $53.6 \%$ in SAVOR-TIMI 53 to $81.0 \%$ in EMPA-REG OUTCOME for ACEis. Differences in statin use, aspirin use, HbA1c values, and concomitant medications (metformin, sulphonylurea, and insulin) across the trials were modest.

The included studies formed a small connected network allowing for the comparison of empagliflozin to two other active treatments. This network was star-shaped and comprised three treatment nodes which were connected through the placebo node (Fig. 2). No treatment was examined in more than one trial, resulting in a single trial per treatment arm. No trials examined multiple treatments, resulting in a network based only on direct comparisons.

During the systematic literature review, heterogeneity between the trials was assessed qualitatively, but was not assessed with any formal statistical tests. Standard statistical measures of heterogeneity $\left(I^{2}\right.$ and $\left.Q\right)$ would not provide valid results because of the small number of trials included and the lack of replication on each treatment arm. Additionally, since all trials included only one treatment versus placebo and the evidence network does not contain closed loops, conventional meta-analysis 


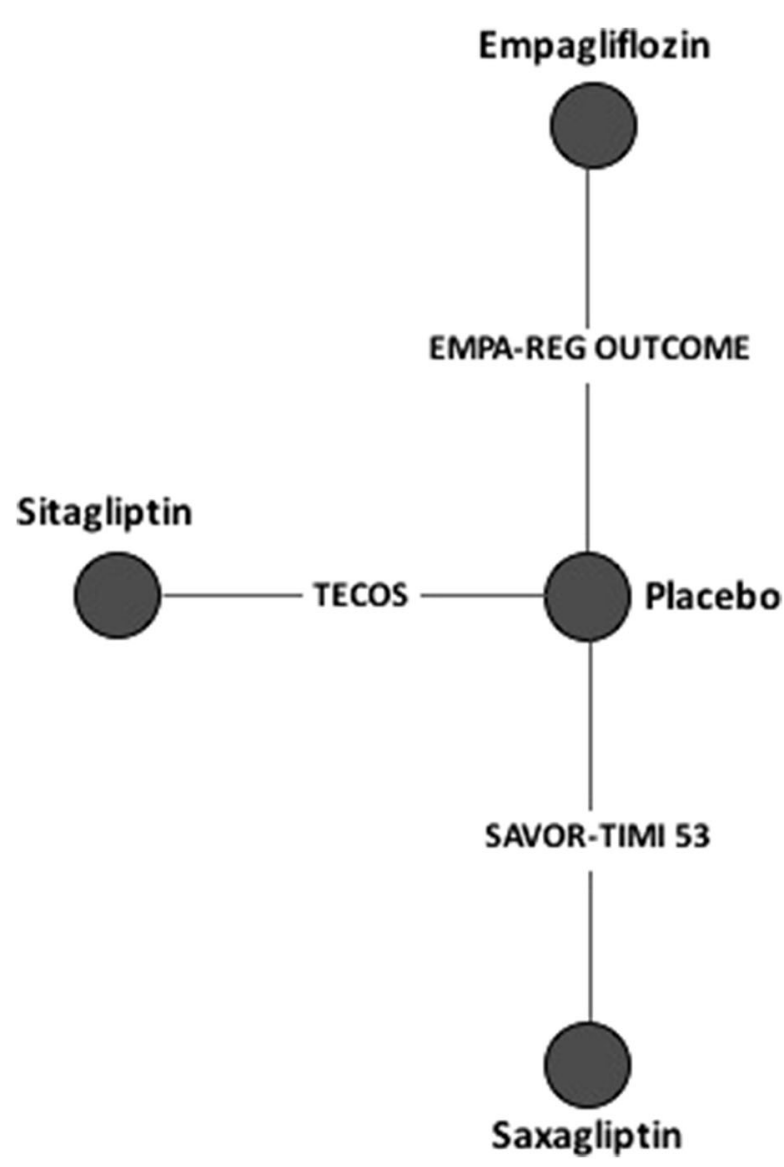

Fig. 2 Overall evidence network

(e.g. pairwise meta-analysis) and assessments of consistency between direct and indirect evidence for the same treatment contrast were not possible. Furthermore, this lack of replication violates the assumptions required for randomeffects models. As a result, only fixed-effects models were run.

\section{Indirect Treatment Comparisons}

The results of indirect treatment comparisons of empagliflozin, saxagliptin, and sitagliptin by a proportional hazards model are presented numerically in Table S9 of the ESM and graphically in Fig. 3. Hazard ratios from individual studies comparing active interventions to placebo are presented in Table S10 of the ESM.

Empagliflozin showed a statistically significantly lower risk of cardiovascular-related mortality compared to saxagliptin (HR 0.60; 95\%
CrI 0.46-0.80) and sitagliptin (HR 0.60; 95\% CrI 0.46-0.79). Empagliflozin also showed a significantly lower risk for all-cause mortality than both saxagliptin (HR 0.61; 95\% CrI 0.49-0.76) and sitagliptin (HR 0.67; 95\% CrI 0.54-0.83).

Further, empagliflozin showed a nonsignificant difference in risk for the composite endpoint (cardiovascular-related mortality, nonfatal myocardial infarction and nonfatal stroke in all trials) versus saxagliptin and sitagliptin.

Empagliflozin also showed a significantly lower risk for hospitalization due to heart failure when compared to both saxagliptin (HR 0.51; 95\% CrI 0.37-0.70) and sitagliptin (HR 0.65; 95\% CrI 0.47-0.90).

Treatment with empagliflozin showed a nonsignificant difference in risk for nonfatal stroke when compared to saxagliptin (HR 1.12; 95\% CrI 0.76-1.63); but these calculations were not adjusted for the mortality benefit of empagliflozin. Additionally, a nonsignificant difference in risk for nonfatal myocardial infarction was observed for treatment with empagliflozin when compared to saxagliptin (HR 0.92; 95\% CrI 0.69-1.20).

It was not feasible to use meta-regression techniques to adjust for differences in patient characteristics across trials. As the network of evidence consisted only of treatments informed by a single trial, there were not enough data to adequately assess the effects of these covariates.

\section{DISCUSSION}

This indirect treatment comparison by NMA resulted in the inclusion of three RCTs, and derived the estimates for comparison between empagliflozin, sitagliptin and saxagliptin. As these glucose-lowering drugs were never compared directly, only direct comparisons of the drugs versus placebo from the individual RCTs were available to inform risk management of cardiovascular events in T2DM. Our results show that treatment of T2DM patients with empagliflozin has a lower risk of cardiovascular deaths, all-cause deaths, and hospitalizations for heart failure compared to treatment with saxagliptin or sitagliptin. Empagliflozin was also 


$\begin{array}{ll}\text { Comparison } & \mathrm{HR}(95 \% \mathrm{Crl}) \\ \text { Empagliflozin vs. Placebo } & 0.62(0.49,0.78) \\ \text { Empagliflozin vs. Saxagliptin } & 0.60(0.46,0.80) \\ \text { Empagliflozin vs. Sitagliptin } & 0.60(0.46,0.79) \\ & \\ \text { Comparison } & \mathrm{HR}(95 \% \mathrm{Crl}) \\ \text { Empagliflozin vs. Placebo } & 0.68(0.57,0.81) \\ \text { Empagliflozin vs. Saxagliptin } & 0.61(0.49,0.76) \\ \text { Empagliflozin vs. Sitagliptin } & 0.67(0.54,0.83) \\ & \\ \text { Comparison } & \mathrm{HR}(95 \% \mathrm{Crl}) \\ \text { Empagliflozin vs. Placebo } & 0.86(0.75,0.99) \\ \text { Empagliflozin vs. Saxagliptin } & 0.86(0.72,1.03) \\ \text { Empagliflozin vs. Sitagliptin } & 0.87(0.73,1.04) \\ & \\ \text { Comparison } & \mathrm{HR}(95 \% \mathrm{Crl}) \\ \text { Empagliflozin vs. Placebo } & 0.65(0.49,0.84) \\ \text { Empagliflozin vs. Saxagliptin } & 0.51(0.37,0.70) \\ \text { Empagliflozin vs. Sitagliptin } & 0.65(0.47,0.90) \\ & \\ \text { Comparison } & \mathrm{HR}(95 \% \mathrm{Crl}) \\ \text { Empagliflozin vs. Placebo } & 1.24(0.92,1.66) \\ \text { Empagliflozin vs. Saxagliptin } & 1.12(0.76,1.63) \\ & \\ \text { Comparison } & \mathrm{HR}(95 \% \mathrm{Crl}) \\ \text { Empagliflozin vs. Placebo } & 0.87(0.70,1.09) \\ \text { Empagliflozin vs. Saxagliptin } & 0.92(0.69,1.20) \\ & \end{array}$

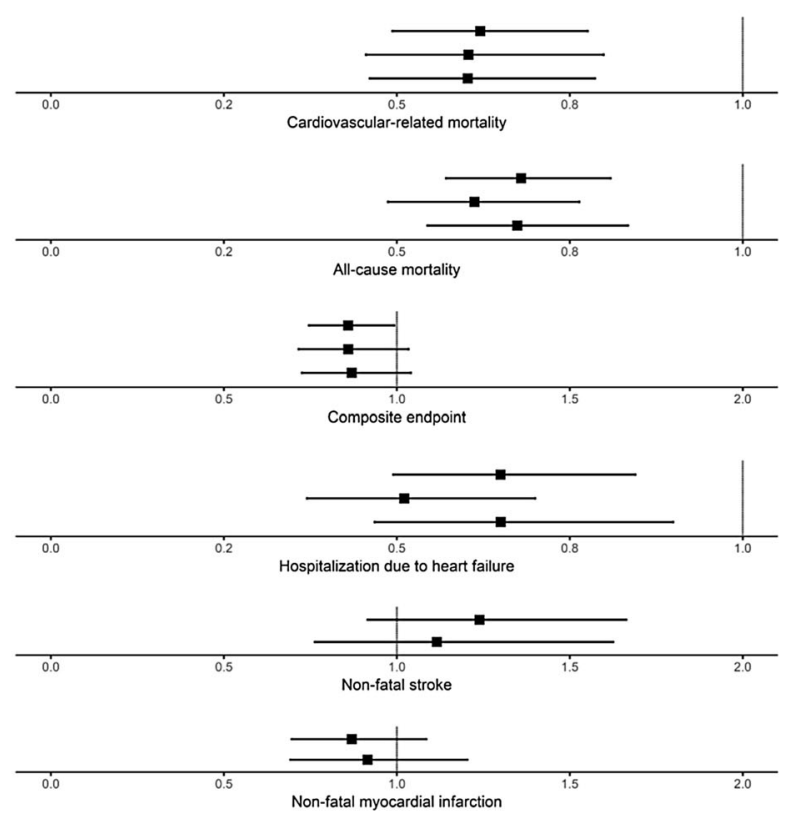

Fig. 3 Plots presenting indirect comparisons of empagliflozin versus other glucose-lowering drugs

associated with nonsignificant improvements over saxagliptin and sitagliptin for nonfatal myocardial infarction and the composite endpoint (cardiovascular-related mortality, nonfatal myocardial infarction and nonfatal stroke).

Previous systematic reviews and pooled analyses that looked at the cardiovascular safety of DPP-4 inhibitors have yielded mixed results [21-26]. Although the majority of these analyses were mainly based on studies conducted in T2DM patients treated for a short duration, the results led to questions about the long-term cardiovascular safety of DPP-4 inhibitors. Wu et al. studied the effect of DPP-4 inhibitors on cardiovascular safety from 50 RCTs with a follow-up duration of at least 24 weeks and observed that cardiovascular-related mortality and incidence of acute coronary syndrome were not significantly different between DPP-4 inhibitors and the comparators [27]. Similarly, a meta-analysis conducted by Savarese et al. including 94 clinical trials showed that DPP-4 inhibitors had no significant effect on cardiovascular-related mortality or stroke [28]. The clinical trials Cardiovascular and Renal Microvascular Outcome Study With Linagliptin in Patients With Type 2 Diabetes Mellitus
(CARMELINA) and Cardiovascular Outcome Study of Linagliptin Versus Glimepiride in Patients With Type 2 Diabetes (CAROLINA), which will be reported soon, focus on cardiovascular outcomes for the DPP-4 agent linagliptin [29]. The results of these two trials will add more information to the existing evidence and help us to better understand the cardiovascular safety of DPP-4 inhibitors. Similarly, analyses of the SGLT-2 inhibitor class have shown a reduction in the risk of adverse cardiovascular events as compared with placebo. As of now, however, there has been no RCT directly comparing active interventions from the SGLT-2 inhibitor and DPP-4 inhibitor classes. In order to address this gap, the present indirect treatment comparison was conducted.

The results observed in this analysis can be primarily attributed to the cardiovascular risk reduction of empagliflozin. Previous works have suggested that treatment with empagliflozin may involve reductions in traditional risk factors as well as other mechanisms that potentially include metabolic and haemodynamic effects [30-34]. Also, studies conducted earlier suggest that there are no significant differences within the class of SGLT-2 inhibitors in their 
abilities to reduce glucose, weight and blood pressure [35], but it remains unknown if the results regarding cardiovascular-related outcomes observed with empagliflozin will also be observed with other SGLT-2 inhibitors. The recently published Canagliflozin Cardiovascular Assessment Study (CANVAS) showed a significantly lower risk for the primary composite endpoint (cardiovascular-related death, nonfatal myocardial infarction, nonfatal stroke) with canagliflozin compared to placebo, but the risk for all-cause mortality and cardiovascular mortality was not significantly different between canagliflozin and placebo [36]. The results from the Multicenter Trial to Evaluate the Effect of Dapagliflozin on the Incidence of Cardiovascular Events (DECLARE), which is evaluating dapagliflozin, another SGLT-2 inhibitor, will also help us to better understand the cardiovascular effects of SGLT-2 drugs [37].

The results of this NMA have some limitations. First, the included RCTs differed with respect to their sample sizes and follow-up periods, and as such, the strength of evidence supporting each intervention and the indirect comparisons is variable. The precision of the estimates may be negatively impacted by the use of only three RCTs in the NMA. Further, in some cases, the included trials differed on clinically meaningful patient characteristics. Formal methods to control for these disparities were not feasible because the evidence base consisted of only one study per treatment. If any characteristic of a particular trial was deemed to be unacceptably different from those observed in other trials, it was suggested that the estimates and comparisons for that treatment should simply be disregarded. As such, the EXAMINE trial was excluded because a patient inclusion criterion for that trial-that a patient must have had an acute coronary syndrome within 15-90 days before randomization-gave the study population an unacceptably different baseline risk as compared with the included trials. Furthermore, it should be noted that the active interventions were not compared to placebo but to the standard of care for the patients, which can vary between trial populations.

\section{CONCLUSIONS}

Treatment with empagliflozin showed a lower risk of cardiovascular-related mortality, allcause mortality and hospitalization due to heart failure compared to the DPP-4 inhibitors saxagliptin and sitagliptin.

\section{ACKNOWLEDGEMENTS}

Authorship. All named authors meet the International Committee of Medical Journal Editors (ICMJE) criteria for authorship for this article, take responsibility for the integrity of the work as a whole, and have given their approval for this version to be published.

Disclosures. Chakrapani Balijepalli is an employee of Precision Xtract, Vancouver, Canada. Rohan Shirali is an employee of Precision Xtract, Vancouver, Canada. Eric Druyts is an employee of Precision Xtract, Vancouver, Canada. Prashanth Kandaswamy Lund is an employee of Boehringer Ingelheim $\mathrm{GmbH}$, Ingelheim, Germany. Anastasia Ustyugova is an employee of Boehringer Ingelheim $\mathrm{GmbH}$, Ingelheim, Germany. Egon Pfarr is an employee of Boehringer Ingelheim $\mathrm{GmbH}$, Ingelheim, Germany. Søren S. Lund is an employee of Boehringer Ingelheim $\mathrm{GmbH}$, Ingelheim, Germany.

Compliance with Ethics Guidelines. This article is based on previously conducted studies and does not contain any studies with human participants or animals performed by any of the authors.

Data Availability. All data generated or analyzed during this study are included in this published article or as supplementary information files.

Open Access. This article is distributed under the terms of the Creative Commons Attribution-NonCommercial 4.0 International License (http://creativecommons.org/licenses/ by-nc/4.0/), which permits any 
noncommercial use, distribution, and reproduction in any medium, provided you give appropriate credit to the original author(s) and the source, provide a link to the Creative Commons license, and indicate if changes were made.

\section{REFERENCES}

1. International Diabetes Federation. IDF diabetes, 8th edn. Brussels: International Diabetes Federation; 2017. http://www.diabetesatlas.org/. Accessed 20 Mar 2018.

2. Huxley R, Barzi F, Woodward M. Excess risk of fatal coronary heart disease associated with diabetes in men and women: meta-analysis of 37 prospective cohort studies. BMJ. 2006;332(7533):73-8.

3. Sloan FA, Bethel MA, Ruiz D Jr, Shea AM, Feinglos $\mathrm{MN}$. The growing burden of diabetes mellitus in the US elderly population. Arch Intern Med. 2008;168(2):192-9 (discussion 9).

4. Meigs JB. Epidemiology of cardiovascular complications in type 2 diabetes mellitus. Acta Diabetol. 2003;40(Suppl 2):S358-61.

5. Centers for Disease Control and Prevention. National diabetes fact sheet: general information and national estimates on diabetes in the United States, 2007. Atlanta: Centers for Disease Control and Prevention; 2007.

6. Centers for Disease Control and Prevention. National diabetes statistics report, 2017: estimates of diabetes and its burden in the United States. Atlanta: Centers for Disease Control and Prevention; 2017.

7. Inzucchi SE, Bergenstal RM, Buse JB, Diamant M, Ferrannini E, Nauck $M$, et al. Management of hyperglycemia in type 2 diabetes, 2015: a patientcentered approach: update to a position statement of the American Diabetes Association and the European Association for the Study of Diabetes. Diabetes Care. 2015;38(1):140-9.

8. Ryden L, Grant PJ, Anker SD, Berne C, Cosentino F, Danchin N, et al. ESC Guidelines on diabetes, prediabetes, and cardiovascular diseases developed in collaboration with the EASD: the Task Force on Diabetes, Pre-diabetes, and Cardiovascular Diseases of the European Society of Cardiology (ESC) and developed in collaboration with the European Association for the Study of Diabetes (EASD). Eur Heart J. 2013;34(39):3035-87.
9. Skyler JS, Bergenstal R, Bonow RO, Buse J, Deedwania $\mathrm{P}$, Gale EA, et al. Intensive glycemic control and the prevention of cardiovascular events: implications of the ACCORD, ADVANCE, and VA diabetes trials: a position statement of the American Diabetes Association and a scientific statement of the American College of Cardiology Foundation and the American Heart Association. Diabetes Care. 2009;32(1):187-92.

10. Udell JA, Cavender MA, Bhatt DL, Chatterjee S, Farkouh ME, Scirica BM. Glucose-lowering drugs or strategies and cardiovascular outcomes in patients with or at risk for type 2 diabetes: a meta-analysis of randomised controlled trials. Lancet Diabetes Endocrinol. 2015;3(5):356-66.

11. Center for Drug Evaluation and Research. Guidance for industry: diabetes mellitus-evaluating cardiovascular risk in new antidiabetic therapies to treat type 2 diabetes. Silver Spring: Center for Drug Evaluation and Research; 2008.

12. European Medicines Agency. Guideline on clinical investigation of medicinal products in the treatment of diabetes mellitus. London: European Medicines Agency; 2010.

13. Zinman B, Wanner C, Lachin JM, Fitchett D, Bluhmki E, Hantel S, et al. Empagliflozin, cardiovascular outcomes, and mortality in type 2 diabetes. N Engl J Med. 2015;373(22):2117-28.

14. Green JB, Bethel MA, Armstrong PW, Buse JB, Engel SS, Garg J, et al. Effect of sitagliptin on cardiovascular outcomes in type 2 diabetes. $\mathrm{N}$ Engl J Med. 2015;373(3):232-42.

15. Pfeffer MA, Claggett B, Diaz R, Dickstein K, Gerstein HC, Kober LV, et al. Lixisenatide in patients with type 2 diabetes and acute coronary syndrome. N Engl J Med. 2015;373(23):2247-57.

16. Scirica BM, Bhatt DL, Braunwald E, Steg PG, Davidson J, Hirshberg B, et al. Saxagliptin and cardiovascular outcomes in patients with type 2 diabetes mellitus. N Engl J Med. 2013;369(14):1317-26.

17. White WB, Cannon CP, Heller SR, Nissen SE, Bergenstal RM, Bakris GL, et al. Alogliptin after acute coronary syndrome in patients with type 2 diabetes. N Engl J Med. 2013;369(14):1327-35.

18. Dias S, Sutton AJ, Ades AE, Welton NJ. Evidence synthesis for decision making 2: a generalized linear modeling framework for pairwise and network meta-analysis of randomized controlled trials. Med Decis Mak. 2012;33(5):607-17.

19. Lunn D, Spiegelhalter D, Thomas A, Best N. The BUGS project: evolution, critique and future directions. Stat Med. 2009;28(25):3049-67. 
20. Spiegelhalter D, Thomas A, Best N, Lunn D. WinBUGS user manual v.1.4. Cambridge: Medical Research Council Biostatistics Unit; 2003.

21. Engel SS, Golm GT, Shapiro D, Davies MJ, Kaufman KD, Goldstein BJ. Cardiovascular safety of sitagliptin in patients with type 2 diabetes mellitus: a pooled analysis. Cardiovasc Diabetol. 2013;12:3.

22. Schweizer A, Dejager S, Foley JE, Couturier A, Ligueros-Saylan M, Kothny W. Assessing the cardiocerebrovascular safety of vildagliptin: meta-analysis of adjudicated events from a large phase III type 2 diabetes population. Diabetes Obes Metab. 2010;12(6):485-94.

23. White WB, Pratley R, Fleck P, Munsaka M, Hisada $\mathrm{M}$, Wilson $\mathrm{C}$, et al. Cardiovascular safety of the dipetidyl peptidase- 4 inhibitor alogliptin in type 2 diabetes mellitus. Diabetes Obes Metab. 2013;15(7):668-73.

24. Monami M, Ahren B, Dicembrini I, Mannucci E. Dipeptidyl peptidase-4 inhibitors and cardiovascular risk: a meta-analysis of randomized clinical trials. Diabetes Obes Metab. 2013;15(2):112-20.

25. Frederich R, Alexander JH, Fiedorek FT, Donovan $\mathrm{M}$, Berglind $\mathrm{N}$, Harris $\mathrm{S}$, et al. A systematic assessment of cardiovascular outcomes in the saxagliptin drug development program for type 2 diabetes. Postgrad Med. 2010;122(3):16-27.

26. Johansen OE, Neubacher D, von Eynatten M, Patel S, Woerle HJ. Cardiovascular safety with linagliptin in patients with type 2 diabetes mellitus: a prespecified, prospective, and adjudicated meta-analysis of a phase 3 programme. Cardiovasc Diabetol. 2012;11:3.

27. Wu S, Hopper I, Skiba M, Krum H. Dipeptidyl peptidase-4 inhibitors and cardiovascular outcomes: meta-analysis of randomized clinical trials with 55,141 participants. Cardiovasc Ther. 2014;32(4):147-58.

28. Savarese G, Perrone-Filardi P, D'Amore C, Vitale C, Trimarco B, Pani L, et al. Cardiovascular effects of dipeptidyl peptidase- 4 inhibitors in diabetic patients: a meta-analysis. Int $\mathrm{J}$ Cardiol. 2015;181:239-44.
29. Rosenstock J, Marx N, Neubacher D, Seck T, Patel S, Woerle HJ, et al. Cardiovascular safety of linagliptin in type 2 diabetes: a comprehensive patient-level pooled analysis of prospectively adjudicated cardiovascular events. Cardiovasc Diabetol. 2015;14:57.

30. Ferrannini E, Mark M, Mayoux E. CV protection in the EMPA-REG OUTCOME trial: a "thrifty substrate" hypothesis. Diabetes Care. 2016;39(7):1108-14.

31. Fitchett D, Zinman B, Wanner C, Lachin JM, Hantel $S$, Salsali A, et al. Heart failure outcomes with empagliflozin in patients with type 2 diabetes at high cardiovascular risk: results of the EMPA-REG OUTCOME® trial. Eur Heart J. 2016;37(19):1526-34.

32. Ryden L, Shahim B, Mellbin L. Clinical implications of cardiovascular outcome trials in type 2 diabetes: from DCCT to EMPA-REG. Clin Ther. 2016;38(6):1279-87.

33. Sattar N, McLaren J, Kristensen SL, Preiss D, McMurray JJ. SGLT2 Inhibition and cardiovascular events: why did EMPA-REG outcomes surprise and what were the likely mechanisms? Diabetologia. 2016;59(7):1333-9.

34. Swedberg K, Ryden L. Treatment of diabetes and heart failure: joint forces. Eur Heart J. 2016;37(19):1535-7.

35. Abdul-Ghani M, Del Prato S, Chilton R, DeFronzo RA. SGLT2 inhibitors and cardiovascular risk: lessons learned from the EMPA-REG OUTCOME study. Diabetes Care. 2016;39(5):717-25.

36. Neal B, Perkovic V, Mahaffey KW, de Zeeuw D, Fulcher G, Erondu N, et al. Canagliflozin and cardiovascular and renal events in type 2 diabetes. N Engl J Med. 2017;377(7):644-57.

37. National Institutes of Health. Multicenter trial to evaluate the effect of dapagliflozin on the incidence of cardiovascular events (DECLARE-TIMI58). ClinicalTrials.gov. 2016. https://clinicaltrials.gov/ct2/ show/NCT01730534. Cited 19 Dec 2016. 